\title{
Pulmonary tuberculosis masquerading as broncho- genic carcinoma with lymphangitic spread of tumor
}

\author{
Chaitanya Ahuja ${ }^{1}$, Ranjit J ain ${ }^{1}$, Takeshwar Tiwari $^{2}$, Shilpa J ain ${ }^{1}$, Meghna Chadha ${ }^{1}$ \\ 1. Wayne State University, Detroit Medical Center, United States. 2. Brooklyn Hospital Center, United States. \\ Correspondence: Chaitanya Ahuja. Address: Wayne State University, Detroit Medical Center, United States. Email: \\ drchaitanyaahuja@gmail.com
}

Received: March 2, 2012

Accepted: May 23, 2012

Published: December 1, 2012

DOI : $10.5430 /$ jbgc.v2n2p115

URL: http://dx.doi.org/10.5430/jbgc.v2n2p115

\begin{abstract}
Pulmonary tuberculosis may present as a mass like lesion, which may mimic neoplasm. We report the case of a middle-aged lady presenting with cough, intermittent fever, hemoptysis and weight loss. A large non-cavitating mass lesion with thickening of the surrounding interlobular septa and adjacent right major fissure was demonstrated on CT scan. A diagnosis of bronchogenic carcinoma with lymphangitic spread of tumor was made on imaging appearance. Image guided biopsy confirmed tuberculosis. We reviewed the existing literature.
\end{abstract}

\section{Key words}

Pulmonary tuberculosis, Lung cancer, Lymphangitis carcinomatosis

\section{I ntroduction}

A total of 9.2 million cases of tuberculosis are reported worldwide annually and the disease continues to be a common health problem despite efforts at eradication and control. Although the incidence of tuberculosis and tuberculosis-related mortality has been on the decline in the United States, with an incidence rate of 4.6 per 100,000 people, it remains a problem among the migrant and HIV-infected populations.

Pulmonary tuberculosis, among other chronic infections, may simulate or co-exist with bronchogenic neoplasms on imaging studies. Tuberculomas can appear masslike and tend to be mistaken for malignancy if typical characteristics, such as upper lobe involvement and calcification, are absent. The most common clinical manifestations are cough and chest pain, and the most common radiographic finding was a solitary pulmonary nodule. However, pulmonary tuberculosis mimicking lung cancer with lymphangitic spread of tumor, has not been previously cited in medical literature. The clinical history of hemoptysis and weight loss may also be misleading, since this maybe the presenting symptom of both tuberculosis and lung cancer.

When the combination of clinical, laboratory, and imaging findings does not help to exclude malignancy, transthoracic needle biopsy can to lead to a timely diagnosis. This helps to initiate appropriate therapy. 


\section{Case report}

A 42-year-old lady presented with a 3-month history of cough, intermittent fever, occasional bouts of hemoptysis and significant weight loss. She was a non-smoker and her immune status was normal. Initial chest radiograph showed consolidation in right upper lobe, which did not respond to routine course of antibiotics. Further evaluation with CT scan demonstrated a $6 \mathrm{~cm}$ mass with irregular margins, in the right upper lobe, without cavitation or calcification. The surrounding lung showed thickening of the interlobular septa and the adjacent major fissure (see Figures A-C). Prominent lymph nodes were seen at the right hilum. There was no pleural effusion. With these imaging appearances, a provisional diagnosis of bronchogenic carcinoma with lymphangitic spread was made. The patient underwent a CT guided biopsy (see Figure D). The histopathological examination revealed granulomas and acid fast bacilli, confirming tuberculosis. The patient responded well to antitubercular therapy.
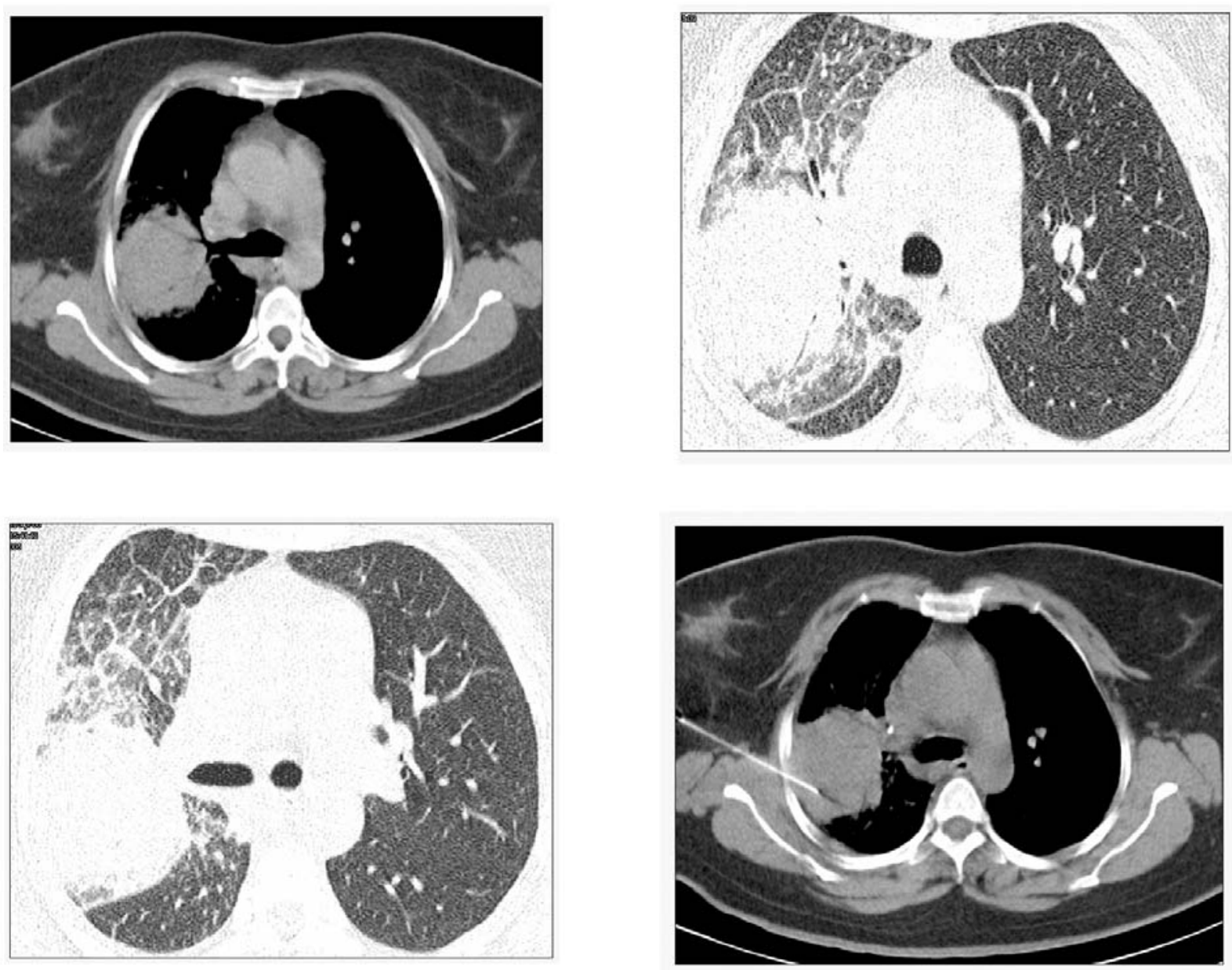

Figure. 42 year-old woman who was referred for suspected lung cancer.

A: An axial CT image in a mediastinal window setting demonstrates a large mass in the right upper lobe with no cavitation or calcification. Prominent lymph node was seen at the right hilum and another image showed hilar calcification.

B \& C: Axial Thin-section CT at lung window setting demonstrates a large mass in the right upper lobe with thickening of the interlobular septa and major fissure, which is predominantly smooth. Intralobular vessels appear slightly prominent indicating centrilobular interstitial thickening. There is no associated lung architectural distortion.

D: Axial CT image in a mediastinal window setting demonstrates transthoracic needle biopsy of the mass. The pathological diagnosis was tuberulosis. 


\section{Discussion}

Tuberculosis often mimics other diseases including malignancy, and has been rightly called a benign imposter ${ }^{[1]}$. In a retrospective analysis by Rolston et al, tuberculosis accounted for $27 \%$ of all infections, initially presumed to be lung cancer on imaging studies. Fungal infections accounted for $46 \%$ of these infections ${ }^{[2]}$.

Radiological manifestations of tuberculosis are varied and dependent on several factors, including prior exposure to tuberculosis, age, and immune status of the patient. Lymphadenopathy is the radiological hallmark of primary tuberculosis. The right paratracheal and right hilar lymph nodes are more commonly involved. Parenchymal involvement more commonly takes the form of homogenous consolidation, although patchy, linear, nodular and mass-like forms are also seen. Pleural effusion may be present ${ }^{[3]}$.

Postprimary tuberculosis may present with consolidation, particularly in apical and posterior segments of the upper lobes; cavitation being the hallmark of disease. Tuberculomas may be seen as sharply marginated lesions measuring between $0.5 \mathrm{~cm}-4 \mathrm{~cm}$. Airway involvement may manifest with bronchial stenosis, tree in bud opacities, endobroncial spread of infection and traction bronchiectesis. Pleural effusion may occur. Lymphadenopathy is uncommon ${ }^{[3]}$.

Miliary disease manifests as innumerable non-calcified nodules between 1-3 mm with mild basilar predominance ${ }^{[3]}$.

Pulmonary lymphangitic carcinomatosis refers to the spread of tumor along lymphatic system of the lung, located in the centrilobular and bronchovascular bundles, in the interlobular septa, and in the subpleural region of the lung ${ }^{[4,5]}$. Lymphangitic spread of cancer has been described in a number of primary tumors, the most important being lung, breast, GI and prostatic neoplasms. A number of etiologies have been established to explain the pattern of lymphangitic spread. It is felt that hematogenous metastases deposit in the lungs and then travel centrally along the lymphatics and venous system. In lung cancer, there can be direct extension to the lymphatics, while some tumors do appear to spread from central lymph nodes peripherally into the lymphatic system ${ }^{[4,5]}$.

The characteristic HRCT findings in lymphangitic spread of carcinoma include smooth or nodular peribronchovascular interstitial, interlobular septal and fissural thickening, with preserved lung architecture ${ }^{[5]}$.

Other than lymphangitis carcinomatosis, a spectrum of non-neoplastic disorders such as pulmonary edema, hemorrhage, or veno-occlusive disease, lymphangiomatosis, amyloidosis, pneumonia, alveolar proteinosis may demonstrate smooth peribronchovascular interstitial and interlobular septal thickening ${ }^{[6]}$.

The finding of interlobular septal thickening in tuberculosis has been infrequently described in literature. In a study by Im et al., almost 35 percent of newly diagnosed patients with pulmonary tuberculosis had interlobular septal thickening on $\mathrm{CT}$, which resolved completely after antitubercular chemotherapy. This has been attributed to local increase in lymphatic flow from the underlying exudative process. Impaired lymph drainage due to associated central lymphadenopathy could also be responsible for thickening of the interlobular septa ${ }^{[7]}$.

Our patient had a fairly large mass with interlobular septal thickening in the surrounding lung. The findings were deceptive for lung cancer with lymphangitic spread. Differentiation from lung cancer based on imaging findings alone may be challenging and often ambiguous, thereby necessitating pathological diagnosis.

\section{References}

[1] Tan CH, Kontoyiannis DP, Viswanathan C, Iyer RB. Tuberculosis: A Benign Impostor. AJR. 2010; 194: 555-561. PMid:20173128 http://dx.doi.org/10.2214/AJR.09.3055 
[2] Rolston KV, Rodriguez S, Dholakia N, Whimbey E, Raad I. Pulmonary infections mimicking cancer: a retrospective, three year review. Support Care Cancer. 1997; 5: 90-3. PMid:9069606 http://dx.doi.org/10.1007/BF01262563

[3] Leung AN. Pulmonary Tuberculosis: The Essentials. Radiology. 1999; 210: 307-322. PMid:10207408

[4] Munk PL, Muller NL, Miller RR, Ostrow DN. Pulmonary Lymphangitic Carcinomatosis: CT and Pathologic Findings. Radiology. 1988; 166: 705-709. PMid:3340765

[5] Webb WR, Muller NL, Naidich DP. Disaese Characterized Primaily by Nodular or Reticulonodular Opacities. In: High resolution CT of the Lung. 3rd ed. Lippincott Williams \& Wilkins. 2001; 560-67

[6] Webb WR. Thin-Section CT of the Secondary Pulmonary Lobule: Anatomy and the Image-The 2004 Fleischner Lecture. Radiology. 2006; 239: 322-338. PMid:16543587 http://dx.doi.org/10.1148/radiol.2392041968

[7] Im JG, Itoh H, Shim YS, Lee J H, Ahn J, Han MC, et al. Pulmonary tuberculosis: CT findings—early active disease and sequential change with antituberculous therapy. Radiology. 1993; 186: 653-660. PMid:8430169 